\title{
Non-Nutritive Sweeteners and Their Contradictory Effect on the Control of Energetic and Glycemic Homeostasis
}

\author{
Ana Paula Souza Silva ${ }^{\mathrm{a}}$, Poliana Guiomar Brasiele ${ }^{\mathrm{a}, \mathrm{b}}$, \\ Sheila Cristina Potente Dutra Luquettia
}

\begin{abstract}
Non-nutritive sweeteners (NNSs) gained attention as dietary tools that provide a free sweet taste of energy to foods. However, its health benefits have been questioned. The aim of this review is to present and discuss the effects of NNSs on energy and glycemic homeostasis, as well as the mechanisms involved. Recent studies in animals and humans have shown that consumption of NNSs appears to have a negative effect on the energy and glycemic homeostasis, promoting body weight gain, insulin resistance, and hyperglycemia. The hypotheses proposed to explain these effects are changes in the perception of taste sweet and weakening of the cephalic response, interaction of NNSs with sweet-taste receptors in the intestinal tract, and changes in intestinal microbiota. However, the determinants of energy and glucose homeostasis are diverse, which may explain in part the unclear effects evidenced in many studies. Therefore, other studies are important to clarify the conflicts between the safety of NNSs and metabolic disorders.
\end{abstract}

Keywords: Artificial sweeteners; Non-nutritive sweeteners; Obesity; Weight gain; Insulin resistance

\section{Introduction}

The obesity epidemic which affects different parts of the world and is related to the consumption of highly hypercaloric diets associated with a relative lack of exercise, resulting in energy imbalances, can cause overweight and obesity, stored fat in adipose tissue [1]. The number of individuals with overweight and obesity has increased over the years, and the fundamental cause of this condition is an energy imbalance between calories consumed and calories expended. The increase in BMI accompanies the risk for several chronic non-communicable

Manuscript submitted December 23, 2018, accepted December 31, 2018

aDepartment of Nutrition, Institute of Biological Sciences, Federal University of Juiz de Fora, Juiz de Fora, Minas Gerais, Brazil

${ }^{b}$ Corresponding Author: Poliana Guiomar Brasiel, Department of Nutrition, Institute of Biological Sciences, Federal University of Juiz de Fora, Juiz de Fora, Minas Gerais, Brazil. Email: guidinhabra@hotmail.com

doi: https://doi.org/10.14740/jem547 diseases [2].

The shortages of reliable clinical researches have led to substantial confusion over the ideal combination of diets that can promote a healthy life, especially about the consumption of carbohydrates and fats. The low-fat diet has been at the heart of dietary guidelines for decades. Currently, the controversy over ideal dietary intake revolves around the consumption of simple sugars. The substantial increase in the prevalence of obesity worldwide attributed in part to the excessive consumption of sugars [3].

In an attempt to control the situation presented, small changes in diet to prevent weight gain have been encouraged [3]. In this sense, non-nutritive sweeteners (NNSs), including low-calorie sweeteners, artificial sweeteners and no caloric sweeteners (NNSs: acesulfame-K, aspartame, saccharin and sucralose), have gained attention as dietary tools capable of providing extra energy-free sweet taste to foods and beverages containing caloric sugars, being used in conjunction with other conducts to promote weight loss, glycemic normalization and increase adherence to diet [4-7].

However, since its approval by the Food and Drug Administration (FDA), the non-nutritive artificial sweeteners and its health benefits have been questioned, since they can promote metabolic changes negatively $[8,9]$. Recent studies in animals and humans suggest that the use of artificial sweeteners can affect energy and metabolic homeostasis, inducing weight gain and intolerance to glucose [10-12]. Whether the factors involved in these adverse metabolic effects attributed to the consumption of artificial sweeteners is not yet well defined. However, some mechanisms have been hypothesized. The main ones are changes in the perception of sweet taste and consequently weakening the cephalic response, and functional changes in the composition of the intestinal microbiota and interaction of artificial sweeteners with sweet-taste receptors in the intestine.

Given the high current controversy regarding the use of artificial sweeteners and its health consequences, this study aims to conduct a review of the metabolic effects of these, particularly about the impact on energy and glycemic homeostasis, as well as mechanisms involved in these effects.

\section{Literature Search}

This integrative review was conducted by searching from electronic database MEDLINE, PubMed platform. The descriptors 
"non-nutritive sweeteners", "obesity" and "glucose intolerance" were used in combination.

Original articles with animal models, in vitro studies and with humans, published in English, related to the use of artificial sweeteners and their effects on energetic and glycemic homeostasis were included. For purposes of conceptualization, contextualization of the theme and understanding of some mechanisms were used in review article.

\section{Discussion}

\section{Effects of NNSs in energetic and glycemic homeostasis}

Before the FDA's approval of authorizing the non-nutritive artificial sweeteners, a series of toxicological and clinical studies were conducted in several species, including humans, to demonstrate that the NNSs were safe and well tolerated. Research conducted in subjects with and without diabetes had shown that even at extremely high doses of sucralose or aspartame, there were no evidenced changes in blood glucose, C-peptide or glycated hemoglobin (HbA1c) [13-15].

In contrast, recent data in studies with human and animal models have shown that consumption of NNSs has adverse and controversial effect on the control of energy and glycemic homeostasis, unlike those which have been previously demonstrated [16-21].

Swithers et al [16] evaluated in rats the effects of saccharin sweetener consumption associated with low or high-energy diet (high fat). It showed that unlike the animals fed with low energy diet content and saccharin, the animals, especially females, fed with high calorie and a high-fat diet supplemented with saccharin, showed a significant increase in energy consumption, weight gain, and adiposity. The authors suggested that the most unfavorable consequences of the use of sweeteners can occur in people who most likely use them for weight control, especially in women who consume a "westernized" diet and are already prone to gain excess weight.

A study by Suez et al [17] showed that the consumption of formulations containing NNSs drove the development of glucose intolerance, through the induction of functional change and the composition of the intestinal microbiota. These deleterious metabolic effects of NNSs were reversed by treatment with antibiotics and could be transferable to germ-free mice after fecal transplantation of microbiota mice that received NNSs. Thus, the authors suggest that excessive consumption of NNSs promotes dysbiosis, associated with the development of metabolic changes.

Other studies also showed effects of using NNSs in glucose homeostasis. Studies in lean mice or with obesity induced by a high-fat diet observed that as the lean rats, mice fed with high-fat diet and with sweeteners added water (saccharin or sucralose or aspartame) developed hyperinsulinemia, glucose intolerance and resistance the action of insulin [17-19].

Swithers et al [19] also found that excess exposure to sweeteners interfered with the relationship between sweet taste (sweet test) and calories, and could affect energy balance and suppressed the release of glucagon-like peptide-1 (GLP-1), which could alter glucose homeostasis, reduce satiety and lead to positive energy balance.

Data from four studies conducted in humans reinforce the potential effect of NNSs in the regulation of glucose homeostasis. Acute consumption of NNSs through diet soda or a small amount of sucralose (24 mg in $200 \mathrm{~mL}$ water) just before an oral glucose load significantly increased the secretion of GLP-1 in healthy children, young, and obese or overweight adults [20-22]. Such change cannot be obtained in individuals already with type 2 diabetes [21, 22]. Furthermore, the ingestion of sucralose affects the glycemic response to oral glucose load, being able to increase glycemia and lead to increased insulin secretion in obese individuals [23].

Another factor observed in human studies has been the concentration of glucose-dependent insulin tropic polypeptide (GIP) in plasma, suggesting that acute ingestion of sucralose may lead to insulin resistance. To maintain the same blood glucose; insulin concentrations were $20 \%$ higher than regular levels in obese subjects who ingested sucralose, compared to subjects receiving only water before glucose intake [23]. However some results in lean and healthy adults have shown that sucralose is not able to affect the glycemic response and hormonal [24-27].

Among the many present impasses regarding the safety of NNSs use, it is necessary to understand the potential physiological mechanisms involved, enabling a correct interpretation of data, and then link them; or there are no possible metabolic disorders that may be related to prolonged and repeated use of artificial sweeteners.

\section{Mechanisms involved in energy and glycemic homeostasis associated with sweeteners use}

To establish a cause-effect relationship between using of NNSs and metabolic disorders, several hypotheses were described. Among these are changes in the perception of sweet taste and the consequent weakening of the cephalic response, interaction of artificial sweeteners with sweet-taste receptors in the intestine and alteration in intestinal microbiota.

In the following are those mechanisms that can act in isolation or together.

\section{Influence of artificial sweeteners in the perception of sweet taste and the weakening of the cephalic response}

The attraction to the sweet taste seems to have an innate basis, where there will be a positive response to sweetness even in the absence of previous experience. However, there is evidence that these innate responses are sensitive to conditioning, and can be shaped by experiences in the pre and postnatal period $[28,29]$.

With the advent of NNSs, often there is exposure to the sweet taste in the absence of an energy source; and as a result of this exposure, there may be adjustment or reduction in responsiveness and sensitivity of sweet taste receptor known as T1R2/T1R3 [30, 31]. Such adaptation is mediated, at least in 
part, by physiological changes within cells to neurons projecting to the perception of sweet taste. Given the adjustment, the sensitivity of sweet taste receptors is reduced $[32,33]$.

Oral repeated stimulation with caloric and natural sweeteners, such as glucose and saccharose results in lower responsiveness and intensity perceived the sweet stimulus, both native and artificial one $[30,32]$. According to some studies, the NNSs can produce the perception of sweet taste through highaffinity binding to the heterodimer T1R2/T1R3 [34, 35] at low concentrations. After binding to the G protein-coupled receptor, it is activated by increasing the production of intracellular messengers. This mechanism involves the activation of transducing channel (TRPM5), resulting in increased intracellular calcium, and release of neurotransmitters [36].

The high affinity of NNSs with receptors raise the possibility that their long-term repeated stimulation can increase the sensory adaptation and reduce sensitivity to sweet taste, which in turn may influence the acceptability of caloric and non-caloric sweeteners [30], the weakening of the cephalic response, leading to a dissociation of sweet flavor to the caloric content [10].

Davidson et al [10] in one of the studies supported this hypothesis, where repeated use of saccharin and other NNSs promoted dissociation of sweet taste to caloric content, as they reduced the effectiveness of associations learned between sweet flavor and calories after intake, leading to higher energy consumption, and consequently, biggest weight gain. Thus, although the NNSs do not contribute directly to calories of the diet, its continuous consumption indirectly promotes the higher caloric intake, due to reduced sensitivity to sweet taste, which interferes with food control by cephalic phase.

The sweet taste of foods is one of the principal initiators of the pre-ingestive reflections on the cephalic phase (secretion of hormones of the gastrointestinal tract, thermogenesis and metabolism) [37-39]. Such conditions are designed to anticipate and prepare the TGI for the arrival of nutrients, thereby increasing the efficient use of nutrients and minimizing the extent to which these nutrients alter homeostasis and promote the positive energy balance, which in the long term can lead to significant increases in body weight and food intake [40]. The efficiency of energy regulation is dependent, at least in part, on the recall of the cephalic phase responses and these responses depend on the ability of the sweet taste in the caloric signaling. Then experiences that weaken this signaling, such as in the use of NNSs can also cause disturbances in the control of food intake and body weight $[41,42]$.

Studies in rats subjected to different experiments receiving caloric (glucose) and no caloric sweeteners (acesulfame-K, saccharin), showed that compared to mice always consuming sweetened diet with glucose, those who were fed with NNSs diet presented greater body mass, accumulated more fat, had reduction of response to compensate for calories and had lower thermic effect of food [10, 12, 43, 44]. These effects reinforce the possibility that the continued use of artificial sweeteners is related to the weakening of the cephalic response, possible reduction in receptor response sensitivity and the ability of perception to predict energy and promote autonomic and endocrine responses that prepare the digestive tract to the optimum food intake process, such as cephalic response.
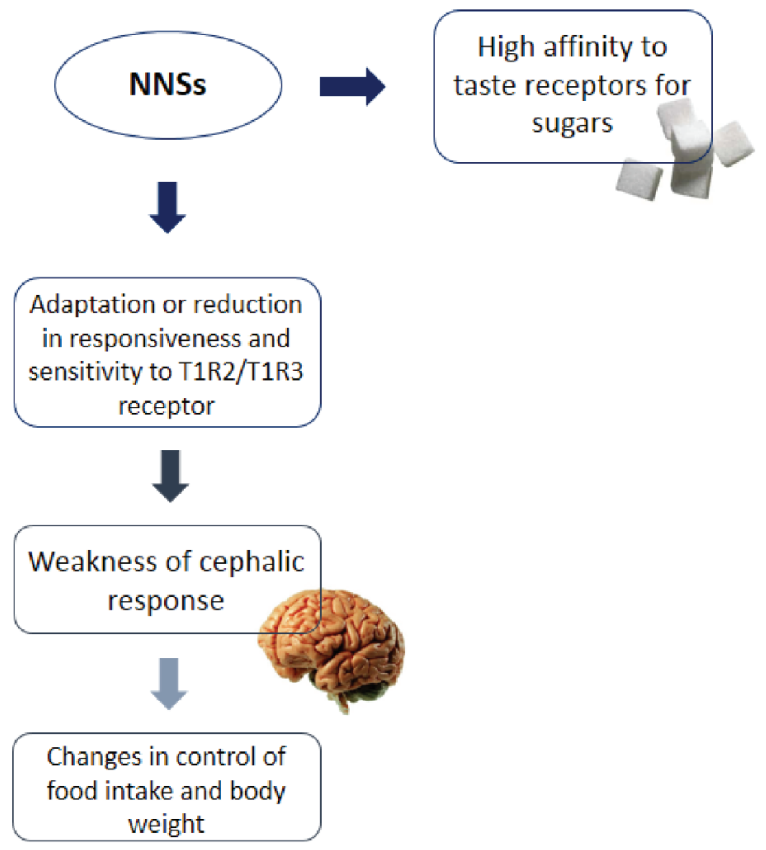

Figure 1. Influence of NNSs in the perception of sweet taste and in the cephalic response.

Also, compared to control rats that received a diet with caloric sweeteners, animals that consumed a diet with NNSs, presented hyperglycemia when underwent glucose tolerance test. Another relevant response to the oral glucose load was the reduction of incretin (GLP-1), which was not observed when glucose was infused directly into the stomach by gavage or preventing the stimulation of fresh flavor orally [19]. These results showed NNSs influencing the glucoregulatory responses when a glucose load was orally administered, but not the same when it was released directly into the stomach. These data reinforce the hypothesis that is breaking the seized answers, triggered by tasting the sweet taste, which alters glucose homeostasis in rodents, not being in that case related to post-feeding behavior consequences. Figure 1 showed the influence of NNSs in the perception of sweet taste and in the cephalic response.

Receptor discovery for the sweet taste in the intestine and their possible interaction with NNSS

The small intestine and colon perform a significant role on satiety via the release of secreted peptides in response to food intake, such as cholecystokinin (CCK), GIP, GLP-1 and polypeptide Y (PYY) [45]. Both lipids and carbohydrates, except fructose, increase the concentration of the hormone GIP. Monosaccharides, particularly glucose, are primarily responsible for stimulating GLP-1 [46]. Recently, it was reported that the presence of taste receptors in different parts of TGI, and since then has been involved in several metabolic processes such as glucose detection, secretion of satiety hormones and glycemic control. Intestinal enterendocrine cells express a series of taste 
transduction molecules, similar to those found in oral mucosa, including T1R2/T1R3 receptors, which is associated with specific protein $\mathrm{G}$, called $\alpha$-gustducin [47].

Experiments that manipulate the signaling pathway involved in sweet taste transduction may provide the considerable evidence of the expression of sweet taste receptors in the gut. Mice without $\alpha$-gustducin or T1R3 showed an inadequate response to incretins when stimulated by glucose $[48,49]$. The incretins (GLP-1, GIP) are intestinal hormones which are released into the blood and stimulate the pancreatic beta cells to secrete insulin and also intervene in the control of appetite and motility [49].

The so-called "incretin effect" was described in 1960, referring to the fact that an oral glucose load causes a significantly higher insulin response to that caused by an intravenous glucose load, even when both are combined to make the same increase in the levels of blood glucose [50]. The assumption that the taste signaling pathways in the intestine can intervene in the "incretin effect" is supported by two observations. The first is that the GLP-1 release is completely blocked by antagonists of sweet taste receptors and $\alpha$-gustducin $[48,51]$. The second is that in knockout mice these receptors glucose homeostasis is significantly unbalanced when they are administered after food intake and when fasted [48].

Besides its significant function of regulating the secretion of GLP-1, signaling pathways in sweet taste may play a crucial importance in the regulation of glucose absorption from the intestinal lumen into enterocytes. The data obtained in rodents suggest that intestinal sweet taste receptors are capable of controlling both the active uptake of glucose by modulating the expression of sodium-dependent glucose transporter (SGLT1) [51], and the absorption of passive glucose by modulating the glucose transporter (GLUT2) in the enterocyte and dietary supplementation of NNSs increasing adiposity and causes hyperinsulinemia and insulin resistance in obese mice by diet $[18,52]$.

In vitro studies have been shown that the application of caloric sweeteners and NNSs in enterendocrine cells can induce the secretion of incretins GLP-1 and GIP through T1R3dependent mechanism $[48,51]$. Also, both natural and artificial sweeteners increase the expression and trafficking of glucose transporters (SGLT-1 and GLUT2) in the membrane of enterocytes through their actions on the sweet taste receptors in the intestine $[51,52]$. We also observed in these studies, the amount of sucralose required for the release of incretin by enterendocrine cell is non-linear, since there are stimulation at low concentrations of sucralose $(0.004 \mathrm{mM}$ to $5 \mathrm{mM})$, but not the same at higher levels $(20 \mathrm{mM})$. This may be explained, at least in part, because doses of sucralose using in the studies were often above the estimated maximum (four to six times), in which intake metabolic effects could not be detected [52].

Knockout mice, which lack $\alpha$-gustducin and T1R3, have been found failed in the regulation of intestinal expression of transporter SGLT1, as well as in glucose uptake capacity when exposed to a high carbohydrate diet (70\% saccharose) [51]. Recent data suggest that the sweet taste receptors may contribute to the incretin response by activating SGLT1 through the signaling process of $\alpha$-gustducin [53]. Thus, the results of studies using $\alpha$-gustducin or T1R3 knockout mice determined that the SGLT1 transporter plays a crucial role in the intestinal absorption of glucose and release of incretin [50].

In humans contradictory results were found on this subject. Several studies reported that oral consumption of an artificially sweetened beverage increases the secretion of GLP-1 in response to the oral glucose tolerance test in healthy subjects [20-22]. However, these studies reported different effects on glycemia. The ingestion of sucralose $(48 \mathrm{mg}$ ) affects the glycemic response to an oral glucose load, being able to increase glycemia and lead to increased insulin secretion in obese individuals [23].

Another factor found in human studies is the increased GIP concentration in plasma of obese individuals who consumed sucralose $(48 \mathrm{mg}$ ). The acute ingestion of sucralose could lead to insulin resistance since insulin concentrations were $20 \%$ higher than the adequate levels in obese individuals who ingested sucralose compared with those who consumed water before glucose intake [23].

However, some results in lean and healthy adults have shown that sucralose is not able to affect the glycemic response, hormonal or glucose intake or other carbohydrates [24-27]. Ford et al [25] wanted to analyze the contributions of oral and post-ingestive mechanisms; they measured hormonal responses to oral ingestion or modified precharge of NNSs. The authors showed that there were no changes in the secretion of intestinal hormones in any of offer of NNSs. Thus, there is a lack of consensus about the glycemic effects of NNSs consumption in humans.

Taste receptors are a new source of studies, although still relatively unexplored, offering a very plausible connection between food preferences, energy modulation, metabolic changes, and body weight. However, we still need more particularly human studies, to a greater understanding of the subject.

\section{Intestinal microbiota}

The intestinal microbiota, including the composition and function, distinguishes between the individuals and is influenced by different of environmental factors as diet [54, 55]. Since 2006, changes in the composition of the intestinal microbiota are no longer associated only with increased susceptibility to infections and immune disorders, and also started to be related to insulin resistance and obesity $[56,57]$. This exists not only in the presence of intestinal bacteria, but in the proportion in which these microbial communities being, which may predispose to obesity, and which it has not related to the role of bacteria in food intake, but its role in the greater extraction of energy from food consumed [57-61].

The first studies on the topic have shown an association between exposure to NNSs and changes in the microbiota $[62,63]$. The results of these studies raised the possibility that NNSs can exert effects on human's health through interactions with intestinal microbiota. The understanding of the mechanisms relating in gut taste and nutrient sensing may represent a different approach to these disorders [64].

Corroborating this hypothesis, Suez et al [17] conducted a major study, with assessments in both mice and humans. In all experiments, there was an association between the consumption of NNS and changes in intestinal microbiota and meta- 
bolic parameters.

In this study, it was found that the use of NNSs promoted glucose intolerance through changes in the intestinal microbiota. The authors showed that treatment of mice with artificial sweeteners saccharin or aspartame or sucralose for 11 weeks resulted in higher levels of glucose than in the control group animals that received no artificial sweeteners. Such metabolic phenotype, at least in saccharin, was also mediated by changes in the intestinal microbiota. To check the influence of microbiota in the metabolic response to saccharin, in subsequent experiments they treated mice (maintained with the diet containing NNSs) with two classes of antibiotics of broad-spectrum and against gram-positive bacteria. After 4 weeks of treatment, the metabolic changes were abolished in mice treated with two classes of antibiotics, suggesting that the effects are dependent on the commensal microbiota and that the NNSs may be capable of interacting with the bacteria and produce an inflammatory response. That in turn, alters the body's ability to properly metabolize glucose, leading insulin resistance and increased blood glucose levels [17].

Furthermore, they demonstrated that when germ-free mice that had never been exposed to saccharin, received fecal transplantation from mice fed with saccharin or microbiota incubated in vitro in the presence of saccharin, in both cases it was shown that saccharin induced hyperglycemia. For confirmation of the effects of NNSs in humans, the authors selected individuals who consumed NNSs for a long time, which was assessed by the validated food frequency questionnaire. The survey found positive relationships between consumption of NNSs and metabolic parameter changes, including increased body mass, waist-hip ratio, fasting glucose levels, glycated hemoglobin concentrations, alanine aminotransferase and alterations in glucose tolerance test [17].

Finally, to identify whether NNSs caused changes in plasma glucose concentrations and their relation to changes in the microbiota, seven healthy young volunteers who were not regular users of NNSs were evaluated. After daily exposure to saccharin sweetener in the maximum amount acceptable to the FDA for 1 week, the glucose tolerance test was assessed daily during this period. The authors showed that usual consumption of saccharin increased the glycemic response in the test of oral glucose tolerance in most of the individuals. Consistent with the results of their study in the animal model, in which the fecal transplantation group (human faeces from individuals who received the sweetener) induced the glucose intolerance in germ-free animals [17]. However, there is a need of careful consideration of these results until they can be replicated with a considerable number of participants and treatment time, using randomized controlled trials; even though these results have introduced an unexpected mechanism and heretofore unexploited by which NNSs can produce adverse metabolic consequences.

\section{Conclusions}

Several studies in literature discussed the metabolic consequences of the consumption of NNSs on human health, which explained through various mechanisms proposed to clarify the inconsistent association between repeated use of NNSs and its effects on energy and glycemic homeostasis. However the determinants of energy and glycemic homeostasis are exceptionally complex and diverse. Moreover, the dosages used and the treatment time are quite variable, which does not allow to conclude what amount of consumption may cause changes in metabolic parameters in humans. These factors justify the inconsistencies found around some studies cited in this review. Thus, it can be concluded that further studies, particularly in humans, are necessary for the clarifications of the conflicts between the safety of artificial sweeteners and metabolic disorders.

Limiting the consumption of any NNS may be the better orientation health. Recognizing natural sweeteners that have positive effects on body weight and metabolism can help to achieve current recommendations for restricting the overconsumption of simple sugars.

\section{Financial Support}

None.

\section{Conflict of Interest}

The authors declare that they have no conflict of interest.

\section{References}

1. Hu FB, Malik VS. Sugar-sweetened beverages and risk of obesity and type 2 diabetes: epidemiologic evidence. Physiol Behav. 2010;100(1):47-54.

2. WHO (World Health Organization) Obesity and overweight (2018). Available from: http://www.who.int/ news-room/fact-sheets/detail/obesity-and-overweight.

3. Mooradian AD, Smith M, Tokuda M. The role of artificial and natural sweeteners in reducing the consumption of table sugar: A narrative review. Clin Nutr ESPEN. 2017; 18:1-8.

4. Benton D. Can artificial sweeteners help control body weight and prevent obesity? Nutr Res Rev. 2005;18(1):6376.

5. Williams CL, Strobino BA, Brotanek J. Weight control among obese adolescents: a pilot study. Int J Food Sci Nutr. 2007;58(3):217-230.

6. Bellisle F, Drewnowski A. Intense sweeteners, energy intake and the control of body weight. Eur J Clin Nutr. 2007;61(6):691-700.

7. Morris D, Cuneo P, Stuart M, et al. High-intensity sweetener, energy and nutrient intakes of overweight women and men participating in a weight-loss program. Nutr Res. 1993;13:123-132.

8. Friedhoff R, Simon JA, Friedhoff AJ. Sucrose solution vs. no-calorie sweetener vs. water in weight gain. J Am Diet Assoc. 1971;59(5):485-486.

9. Blundell JE, Hill AJ. Paradoxical effects of an intense sweet- 
ener (aspartame) on appetite. Lancet. 1986;1(8489):10921093.

10. Davidson TL, Martin AA, Clark K, Swithers SE. Intake of high-intensity sweeteners alters the ability of sweet taste to signal caloric consequences: implications for the learned control of energy and body weight regulation. Q J Exp Psychol (Hove). 2011;64(7):1430-1441.

11. Fowler SP, Williams K, Resendez RG, Hunt KJ, Hazuda HP, Stern MP. Fueling the obesity epidemic? Artificially sweetened beverage use and long-term weight gain. Obesity (Silver Spring). 2008;16(8):1894-1900.

12. Swithers SE, Baker CR, Davidson TL. General and persistent effects of high-intensity sweeteners on body weight gain and caloric compensation in rats. Behav Neurosci. 2009;123(4):772-780.

13. Nehrling JK, Kobe P, McLane MP, Olson RE, Kamath S, Horwitz DL. Aspartame use by persons with diabetes. Diabetes Care. 1985;8(5):415-417.

14. Mezitis NH, Maggio CA, Koch P, Quddoos A, Allison DB, Pi-Sunyer FX. Glycemic effect of a single high oral dose of the novel sweetener sucralose in patients with diabetes. Diabetes Care. 1996;19(9):1004-1005.

15. Baird IM, Shephard NW, Merritt RJ, Hildick-Smith G. Repeated dose study of sucralose tolerance in human subjects. Food Chem Toxicol. 2000;38(Suppl 2):S123-129.

16. Swithers SE, Sample CH, Davidson TL. Adverse effects of high-intensity sweeteners on energy intake and weight control in male and obesity-prone female rats. Behav Neurosci. 2013;127(2):262-274.

17. Suez J, Korem T, Zeevi D, Zilberman-Schapira G, Thaiss $\mathrm{CA}$, Maza O, Israeli D, et al. Artificial sweeteners induce glucose intolerance by altering the gut microbiota. Nature. 2014;514(7521):181-186.

18. Mitsutomi K, Masaki T, Shimasaki T, Gotoh K, Chiba S, Kakuma T, Shibata H. Effects of a nonnutritive sweetener on body adiposity and energy metabolism in mice with diet-induced obesity. Metabolism. 2014;63(1):69-78.

19. Swithers SE, Laboy AF, Clark K, Cooper S, Davidson TL. Experience with the high-intensity sweetener saccharin impairs glucose homeostasis and GLP-1 release in rats. Behav Brain Res. 2012;233(1):1-14.

20. Brown RJ, Walter M, Rother KI. Ingestion of diet soda before a glucose load augments glucagon-like peptide-1 secretion. Diabetes Care. 2009;32(12):2184-2186.

21. Brown RJ, Walter M, Rother KI. Effects of diet soda on gut hormones in youths with diabetes. Diabetes Care. 2012;35(5):959-964.

22. Temizkan S, Deyneli O, Yasar M, Arpa M, Gunes M, Yazici D, Sirikci O, et al. Sucralose enhances GLP-1 release and lowers blood glucose in the presence of carbohydrate in healthy subjects but not in patients with type 2 diabetes. Eur J Clin Nutr. 2015;69(2):162-166.

23. Pepino MY, Tiemann CD, Patterson BW, Wice BM, Klein S. Sucralose affects glycemic and hormonal responses to an oral glucose load. Diabetes Care. 2013;36(9):25302535.

24. Ma J, Chang J, Checklin HL, Young RL, Jones KL, Horowitz M, Rayner CK. Effect of the artificial sweetener, sucralose, on small intestinal glucose absorption in healthy human subjects. Br J Nutr. 2010;104(6):803-806. 25. Ford HE, Peters V, Martin NM, Sleeth ML, Ghatei MA, Frost GS, Bloom SR. Effects of oral ingestion of sucralose on gut hormone response and appetite in healthy normal-weight subjects. Eur J Clin Nutr. 2011;65(4):508513.

26. Wu T, Zhao BR, Bound MJ, Checklin HL, Bellon M, Little TJ, Young RL, et al. Effects of different sweet preloads on incretin hormone secretion, gastric emptying, and postprandial glycemia in healthy humans. Am J Clin Nutr. 2012;95(1):78-83.

27. Wu T, Zhao BR, Bound MJ, Checklin HL, Bellon M, Little TJ, Young RL, et al. Artificial sweeteners have no effect on gastric emptying, glucagon-like peptide-1, or glycemia after oral glucose in healthy humans. Diabetes Care. 2013;36(12):202-203.

28. Beauchamp GK, Mennella JA. Early flavor learning and its impact on later feeding behavior. J Pediatr Gastroenterol Nutr. 2009;48(Suppl 1):S25-30.

29. Ventura AK, Mennella JA. Innate and learned preferences for sweet taste during childhood. Curr Opin Clin Nutr Metab Care. 2011;14(4):379-384.

30. Schiffman SS, Cahn H, Lindley MG. Multiple receptor sites mediate sweetness: evidence from cross adaptation. Pharmacol Biochem Behav. 1981;15(3):377-388.

31. Schiffman SS, Pecore SD, Booth BJ, Losee ML, Carr BT, Sattely-Miller E, Graham BG, et al. Adaptation of sweeteners in water and in tannic acid solutions. Physiol Behav. 1994;55(3):547-559.

32. Tonosaki K, Funakoshi M. Cross-adapted sugar responses in the mouse taste cell. Comp Biochem Physiol A Comp Physiol. 1989;92(2):181-183.

33. Tonosaki K. Cross-adapted salt responses in the mouse taste cell. Brain Res. 1992;574(1-2):338-340.

34. Xu H, Staszewski L, Tang H, Adler E, Zoller M, Li X. Different functional roles of T1R subunits in the heteromeric taste receptors. Proc Natl Acad Sci U S A. 2004;101(39):14258-14263.

35. Farkas A, Hid J. The black agonist-receptor model of high potency sweeteners, and its implication to sweetness taste and sweetener design. J Food Sci. 2011;76(8):S465-468.

36. Chandrashekar J, Hoon MA, Ryba NJ, Zuker CS. The receptors and cells for mammalian taste. Nature. 2006;444(7117):288-294.

37. Teff KL, Devine J, Engelman K. Sweet taste: effect on cephalic phase insulin release in men. Physiol Behav. 1995;57(6):1089-1095.

38. Tordoff MG. How do non-nutritive sweeteners increase food intake? Appetite. 1988;11(Suppl 1):5-11.

39. Bruce DG, Storlien LH, Furler SM, Chisholm DJ. Cephalic phase metabolic responses in normal weight adults. Metabolism. 1987;36(8):721-725.

40. Teff K. Nutritional implications of the cephalic-phase reflexes: endocrine responses. Appetite. 2000;34(2):206213.

41. Woods SC, Ramsay DS. Pavlovian influences over food and drug intake. Behav Brain Res. 2000;110(1-2):175182.

42. Cooling J, Blundell JE. Lean male high- and low-fat phe- 
notypes - different routes for achieving energy balance. Int J Obes Relat Metab Disord. 2000;24(12):1561-1566.

43. Swithers SE, Davidson TL. A role for sweet taste: calorie predictive relations in energy regulation by rats. Behav Neurosci. 2008;122(1):161-173.

44. Swithers SE, Martin AA, Davidson TL. High-intensity sweeteners and energy balance. Physiol Behav. 2010;100(1):55-62.

45. Cummings DE, Overduin J. Gastrointestinal regulation of food intake. J Clin Invest. 2007;117(1):13-23.

46. Herrmann C, Goke R, Richter G, Fehmann HC, Arnold R, Goke B. Glucagon-like peptide-1 and glucose-dependent insulin-releasing polypeptide plasma levels in response to nutrients. Digestion. 1995;56(2):117-126.

47. Laffitte A, Neiers F, Briand L. Functional roles of the sweet taste receptor in oral and extraoral tissues. Curr Opin Clin Nutr Metab Care. 2014;17(4):379-385.

48. Jang HJ, Kokrashvili Z, Theodorakis MJ, Carlson OD, Kim BJ, Zhou J, Kim HH, et al. Gut-expressed gustducin and taste receptors regulate secretion of glucagon-like peptide-1. Proc Natl Acad Sci U S A. 2007;104(38):1506915074 .

49. Kokrashvili Z, Mosinger B, Margolskee RF. T1r3 and alpha-gustducin in gut regulate secretion of glucagon-like peptide-1. Ann N Y Acad Sci. 2009;1170:91-94.

50. McIntyre N, Holdsworth CD, Turner DS. New interpretation of oral glucose tolerance. Lancet. 1964;2(7349):2021.

51. Margolskee RF, Dyer J, Kokrashvili Z, Salmon KS, Ilegems E, Daly K, Maillet EL, et al. T1R3 and gustducin in gut sense sugars to regulate expression of $\mathrm{Na}+$-glucose cotransporter 1. Proc Natl Acad Sci U S A. 2007;104(38):15075-15080.

52. Mace OJ, Affleck J, Patel N, Kellett GL. Sweet taste receptors in rat small intestine stimulate glucose absorption through apical GLUT2. J Physiol. 2007;582(Pt 1):379392.

53. Gorboulev V, Schurmann A, Vallon V, Kipp H, Jaschke A, Klessen D, Friedrich A, et al. Na(+)-D-glucose cotransporter SGLT1 is pivotal for intestinal glucose absorption and glucose-dependent incretin secretion. Diabetes. 2012;61(1):187-196.
54. Muegge BD, Kuczynski J, Knights D, Clemente JC, Gonzalez A, Fontana L, Henrissat B, et al. Diet drives convergence in gut microbiome functions across mammalian phylogeny and within humans. Science. 2011;332(6032):970-974.

55. Claesson MJ, Jeffery IB, Conde S, Power SE, O'Connor EM, Cusack S, Harris HM, et al. Gut microbiota composition correlates with diet and health in the elderly. Nature. 2012;488(7410):178-184.

56. Dakin CL, Small CJ, Batterham RL, Neary NM, Cohen MA, Patterson M, Ghatei MA, et al. Peripheral oxyntomodulin reduces food intake and body weight gain in rats. Endocrinology. 2004;145(6):2687-2695.

57. Mahowald MA, Rey FE, Seedorf H, Turnbaugh PJ, Fulton RS, Wollam A, Shah N, et al. Characterizing a model human gut microbiota composed of members of its two dominant bacterial phyla. Proc Natl Acad Sci U S A. 2009;106(14):5859-5864.

58. Tilg H, Moschen AR, Kaser A. Obesity and the microbiota. Gastroenterology. 2009;136(5):1476-1483.

59. Collado MC, Isolauri E, Laitinen K, Salminen S. Distinct composition of gut microbiota during pregnancy in overweight and normal-weight women. Am J Clin Nutr. 2008;88(4):894-899.

60. Harris K, Kassis A, Major G, Chou CJ. Is the gut microbiota a new factor contributing to obesity and its metabolic disorders? J Obes. 2012;2012:879151.

61. Furet JP, Kong LC, Tap J, Poitou C, Basdevant A, Bouillot JL, Mariat D, et al. Differential adaptation of human gut microbiota to bariatric surgery-induced weight loss: links with metabolic and low-grade inflammation markers. Diabetes. 2010;59(12):3049-3057.

62. Abou-Donia MB, El-Masry EM, Abdel-Rahman AA, McLendon RE, Schiffman SS. Splenda alters gut microflora and increases intestinal p-glycoprotein and cytochrome p-450 in male rats. J Toxicol Environ Health A. 2008;71(21):1415-1429.

63. Anderson RL, Kirkland JJ. The effect of sodium saccharin in the diet on caecal microflora. Food Cosmet Toxicol. 1980;18(4):353-355.

64. Sclafani A. Sweet taste signaling in the gut. Proc Natl Acad Sci U S A. 2007;104(38):14887-14888. 